\title{
Probabilistic Evaluation of a Power System's Reliability and Quality Measures
}

\author{
Badr M. Alshammari \\ Department of Electrical Engineering \\ College of Engineering \\ University of Hail \\ Hail, Saudi Arabia \\ bms.alshammari@uoh.edu.sa
}

\begin{abstract}
Reliability and performance quality measures computed so far are deterministic in nature. They represent one operating state (a snapshot of the system conditions) in which the required demand and generation and transmission capacities are known with $100 \%$ certainty. In this paper a general and coherent formulation is presented, which can be used to account for the randomness associated with the load level as well as the availability of generation and transmission capacities. The general probability formulation can be used to calculate various reliability indices and quality measures. The paper describes the new approach for computing probabilistic evaluation (expected value) of the reliability indices and performance quality measures and presents illustrative applications. The methodology used in this paper constitutes a new line of research in the probabilistic reliability evaluation of a system where the derived system-wide performance quality indices are capable of classifying and exhibitionistic areas of deficiencies, bottlenecks and redundancies in large-scale power grids.
\end{abstract}

Keywords-probabilistic reliability; evaluation; quality; power systems

\section{INTRODUCTION}

The competitive electricity market is subjected to an increasing amount of uncertainties such as the demand forecast uncertainty, electricity price volatility, reliability of the generation groups, economic growth uncertainties, and changing environmental and social impacts on the energy sector [1-2]. In addition to considerations of these uncertainties, planners also need to balance the technical requirement of the system and the requirements from investors who aim at profit maximization [3-5]. There is a general utilization of reliability models throughout the world as the advantages of probabilistic methods over the deterministic approaches of planning, designing, operating and maintaining electric utility systems have clearly been recognized [6]. As has happened with many power system disciplines, the prime interest in system security, adequacy, and reliability has gradually shifted from completing and refining the theoretical basis, through developing suitable computational tools for demonstrating the capability and practicality of the methodologies, to upgrading the computational tools to handle the large-scale nature of present power systems and, finally, to relate various security, quality and reliability indices to the practical concerns of utility engineers and executives regarding supply and/or transmission deficiencies as well as the risk associated with ignoring such deficiencies [7-10]. Methods for computing probabilistic contingency-based reliability and performance quality indices have previously been published in [11-17]. These methods are based on a combined contingency analysis and reliability evaluation scheme which integrates both the contingency effect and its probability of occurrence into one routine of analysis [18-19]. In the current work, similar analysis will be used to compute the expected values of different system reliability and performance quality indices. In this context, a "contingency scenario" or a system "demand level" are regarded, in a more general sense, as a "state", which occurs with certain probability and represents a given demand value and an availability pattern of various capacities in the system. This paper shares the results of a recent major industry supported research and development study in which a novel framework was developed [2] for evaluating performance quality indices associated with power system generation demand balance. In this paper, the optimization technique based on three metaphors (dimensions) representing the relationship between available generation capacities and required demand levels, is presened. The novel formulation presented in this paper can accommodate the randomness associated with the load level and the availability of generation and transmission capacities.

\section{PROBLEM FORMULATION}

\section{A. Power System Network Model}

Let $n_{B}$ be the number of buses in the power network, where $n_{B}=n_{L}+n_{G}$, with $n_{L}$ and $n_{G}$ being the number of load and generator buses respectively. Also, in the network model used, $n_{T}$ is the number of transmission branches (lines and transformers). In order to facilitate the subsequent formulation, it is assumed without loss of generality, that the load buses are numbered as $1,2, \ldots, n_{L}$ followed by generator buses as $n_{L}+1, \ldots$, $n_{L}+n_{G}$, where $n_{L}+n_{G}=n_{B}$. For example, the sample power system shown in Figure 1 has $n_{B}=4, n_{G}=2, n_{L}=2$ and $n_{T}=5$. Now, let $A=\left(n_{B} \times n_{T}\right)$ be the bus incidence matrix representing the connectivity pattern between buses and lines. The entries of $A$ are 0,1 or -1 . Therefore, an element $A_{b t}=1$ if bus $b$ is feeding a transmission branch $t, A_{b t}=-1$ if bus $b$ is fed from a branch $t$, and $A_{b t}=0$ otherwise. In the current analysis, the $A$ matrix is partitioned row-wise into $A_{L}$ and $A_{G}$ associated respectively with load and generator buses. The rows of $A$ (or columns of 
$A^{T}$ ) represent groups of buses while the columns of $A$ (or rows of $A^{T}$ ) represent groups of transmission links. We also note that for practical large-scale networks, the matrix $A$ is extremely sparse.

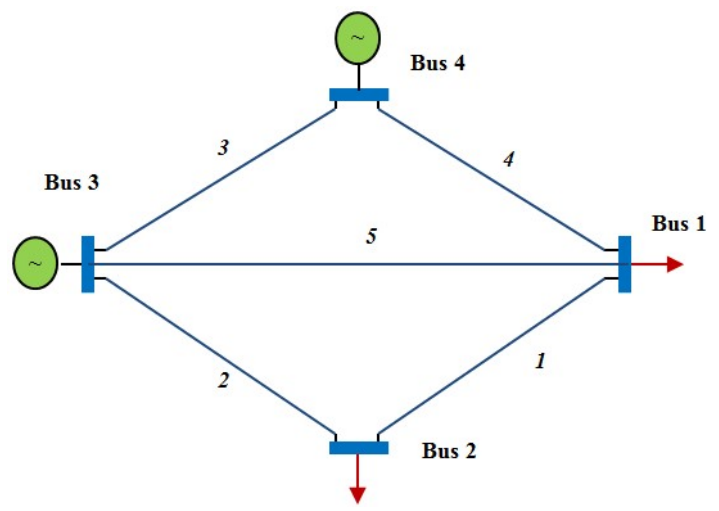

Fig. 1. A sample power system [8]

\section{B. Performance Quality Assessment}

Although the basic definitions pertaining to system performance quality are simple to state and often seem intuitive at first glance, care should be exercised in order to recognize some subtle differences in the definition and formulation of the composite performance quality indices. Let $\bar{P}_{T}$ be a vector of $n_{T}$ elements representing transmission branch capacities, $\bar{P}_{L}$ a vector of $\mathrm{n}_{\mathrm{L}}$ elements of peak bus loads, and $\bar{P}_{G}$ a vector of $n_{G}$ elements representing generator capacities $\bar{P}_{g}$. For simplicity of notation, we shall use $\bar{P}_{T}$ to denote a general element $t$ of the vector $\bar{P}_{T}$ (rather than the more strict notation of $\bar{P}_{T L}$ ). Similarly, we shall use $\bar{P}_{l}$ and $\bar{P}_{g}$ to denote the general elements of $\bar{P}_{L}$ and $\bar{P}_{G}$ respectively. However, when confusion may occur, we will use the strict notation of $\bar{P}_{T i}, \bar{P}_{L i}$ and $\bar{P}_{G i}$. Now, consider the schematic configurations of Figure 2(a) which depicts the transfer connectivity between generations through transmission to load.

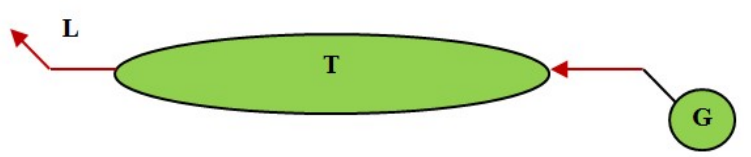

(a)

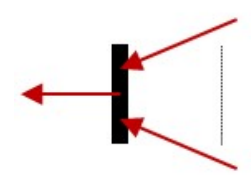

(b)

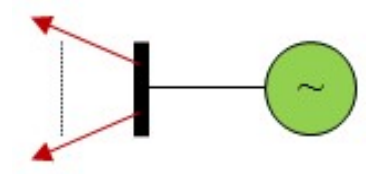

(c)
Fig. 2. G-T-L transfer connectivity [8]

If, for example the local generation capacity $\bar{P}_{g}$ at bus $g$ exceeds the corresponding transmission capability $\sum_{t \in T_{g}} \bar{P}_{t}$ in Figure 2(b), where $T_{g}$ denotes the set of transmission branches connected to generator bus $g$, then using the terminology introduced in the previous section, we may say that a positive amount of $\left(\bar{P}_{g}-\sum_{t \in T_{g}} \bar{P}_{t}\right)$ of generation beyond bus $g$ has been bottled (blocked from usage). We should note that such a definition applies to a specific scenario of system configuration (the $A$-matrix) and loading conditions. For example, in the above discussion, we assumed that the set $T_{g}$ does not represent any of pre-defined contingency scenarios. That is, $T_{g}$ represents the full transmission capacity at bus $g$. In addition to the above definitions, we also define, using similar notation, the following vector for later use: $\overline{\bar{P}}_{G}=$ vector of generation site capacities, which represents the maximum future expanded generation capacity that could be available at the same generation site. The novel framework applied in this paper is based on the original work in [2], in which three dimensions were introduced to represent the relationship between certain system generation capacity and the demand. These tropes relate to the following demand fulfillment issues:

- Need of capacity for demand fulfillment

- Existence of capacity (availability for demand fulfillment)

- Ability of capacity to reach the demand

The first trope defines whether the capacity is needed, the second defines whether the capacity exists, and the last defines whether the capacity can reach (delivered to) the demand. The eight possible combinations associated with the $0 / 1$ (Yes/No) values of the three tropes, are illustrated in Table I. Generation quality indices are defined in terms of the previously defined "1/0" states indicating the (Needed, Exists, Can-reach) true/false values associated with each quality metaphor. We shall use the symbol $Q_{g}$ ijk to indicate the generation quality index state. Also, in the following expressions, we shall use Min $\{x, y, \ldots, z\}$ to indicate the minimum of $x, y, \ldots, z$. The notation $\langle x>$ will be used to denote $\operatorname{Max}\{0, x\}$, which is the maximum of $x$ and zero ( $=x$ if $x>0$, or 0 otherwise).

TABLE I. ILLUSTRATION OF QUALITY ASSESSMENT TROPES

\begin{tabular}{|c|c|c|c|c|}
\hline & \multicolumn{2}{|c|}{$\begin{array}{c}\text { Needed } \\
(\boldsymbol{L}>\mathbf{0})\end{array}$} & \multicolumn{2}{c|}{$\begin{array}{c}\text { Not needed } \\
(\boldsymbol{L}=\mathbf{0})\end{array}$} \\
\hline & Can reach & Cannot reach & Can reach & Cannot reach \\
\hline Exist & Utilized & Bottled & Surplus & Redundant \\
$(C>0)$ & $Q_{g} 111$ & $Q_{g} 110$ & $Q_{g} 011$ & $Q_{g} 010$ \\
\hline Not-Exist & Short-fall & Deficient & Spared & Saved \\
$(C=0)$ & $Q_{g} 101$ & $Q_{g} 100$ & $Q_{g} 001$ & $Q_{g} 000$ \\
\hline
\end{tabular}

Table I summarizes the considered quality indices, namely the Utilized Generation Capacity (Q111), Bottled Generation Capacity (Q110), Shortfall Generation Capacity (Q101), Deficit Generation Capacity (Q100), Surplus Generation Capacity (Q011), Redundant Generation Capacity (Q010), Spared Generation Capacity (Q001), and Saved Generation Capacity (Q000).

\section{Linear Program Formulation}

In the computational scheme of [2], the integrated system quality assessment is performed via solving a master linear programming problem in which a feasible power flow is established which minimizes the total system Load Not Served (LNS) subject to capacity limits and flow equations. The master 
linear program, which utilizes the network bus incidence matrix $\boldsymbol{A}$, is formulated as:

$$
\begin{aligned}
& \text { Objective function }=\left(\text { Minimize } f=\sum_{l=1}^{n_{L}}\left(-P_{l}\right)\right) \\
& \text { with respect to } P_{L}, P_{G} \text {, and } P_{T} \\
& \text { subject to: } \\
& \left.A \cdot P_{T}=\left[\begin{array}{cl}
-\mathbf{P}_{L} \\
P_{G}
\end{array}\right] \begin{array}{l}
P_{L} \leq \overline{P_{L}},-P_{L} \leq 0 \\
P_{G} \leq \overline{P_{G}},-P_{G} \leq 0 \\
P_{T} \leq \overline{P_{T}},-P_{T} \leq \overline{P_{T}}
\end{array}\right\}
\end{aligned}
$$

An optimization software package (CPLEX) has been used to solve the Master Linear Program. The overall process of the evaluation of power systems reliability and quality measures is summarized in the flowchart in Figure 3.

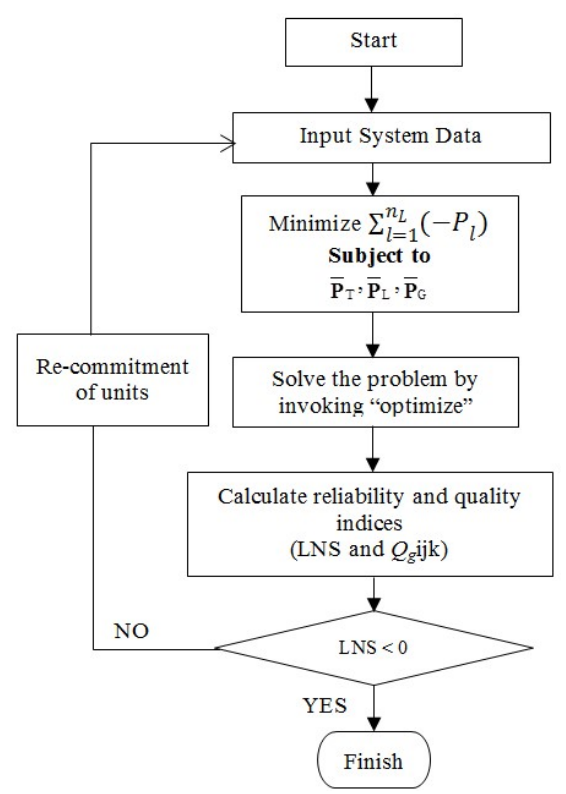

Fig. 3. Flow chart of the proposed methodology

\section{Probabilistic Reliability Indices}

The power system can be described, for the purpose of composite reliability and performance quality assessment, by the three-component model in which generation, transmission, and load are considered as multi-state elements of the power system. For a given operating state $m$, the values of the network variables will be the solution of the maximum load-supply optimization problem described in the previous section. Also, let $f_{m}$ be the probability of operating state $m$ (the sum of $f_{m}$ for all $m$, including base-case scenario is 1). Then, the loss of load may be defined as:

$$
L O L P=\sum_{m=1}^{M_{s}} L O L P^{(m)}
$$

where:

$$
L O L P^{(m)}=\operatorname{Max}_{l}\left\{Y_{l} L O L P_{l}^{(m)}\right\}
$$

represents the system loss of load probability for any operating state $m$ (load levsrl, loss of generation and/or transmission capacities) in the power grid,

$$
L O L P_{l}^{(m)}=\lambda_{l}^{m} f_{m}
$$

represents the loss of load probability at bus $\ell$ for operating state $m$,

$$
\lambda_{l}^{(m)}=\left\{\begin{array}{llll}
0 & \text { if } & P_{l}^{(m)} & \leq P_{l}^{o} \\
1 & \text { if } & P_{l}^{(m)} & >P_{l}^{o}
\end{array}\right.
$$

and $P_{\ell}^{o}$ denotes the scheduled (required) load at load bus $\ell$. Also, in (2), $M_{s}$ denotes the number of all possible states.

\section{Probabilistic EVALUATION}

The reliability and performance quality indices computed so far are deterministic in nature, i.e. they represent one operating state in which the required demand and the generation and transmission capacities are known with $100 \%$ certainty.

\section{A. Proposed Formulation}

In real life, load variations occur randomly and contingencies cause some generation and/or transmission capacities to be lost (become unavailable). In other words, neither the load levels nor the generation or transmission capacities are known with absolute certainty. They are rather subject to random variations and consequently the calculated reliability and performance quality indices are all subject to random variations where only expected values of these indices can be evaluated. For example, the load variations, which are accounted for using the so-called "load-duration curves" can be used to calculate the expected value of the $L N S$, which is widely known as the Expected Load Not-Served (eLNS). On the other hand, the randomness in the generation and transmission capacity availability are accounted for using the so-called forced-outage rates (or availability rates) associated with various facilities. Consequently, the expected values of the performance quality indices $Q_{g 111}, Q_{g 110}, Q_{g 101}$, etc. can be evaluated using the modeled randomness of the system load as well as the generation and transmission capacity availabilities as will be outlined in this section. Methods for computing probabilistic contingency-based reliability and performance quality indices have previously been published [7]. These methods are based on a combined contingency analysis and reliability evaluation scheme which integrates both the contingency effect and its probability of occurrence into one routine of analysis. In the present work, similar analysis will be used to compute the expected values of different system performance quality indices. In this context, a "contingency scenario" or a system "demand level" are regarded, in a more general sense, as a "state" which occurs with certain probability and represents a given demand value and availability pattern of various capacities in the system.

\section{B. Simulation Results}

Consider the sample power system in Figure 4. The system consists of two generators, namely G1 and G2, with a total capacity of $100 \mathrm{MW}$ and connected to two load buses, with a 
total required load of $115 \mathrm{MW}$, via five transmission lines. In this illustrative example, the availability of the two generating units are assumed as 0.9 and 0.8 , respectively (forced outage rates of $\mathrm{G} 1$ and $\mathrm{G} 2$, respectively). Also, for simplicity, all transmission lines are assumed to have availability of 0.85 . The system load is assumed to have three possible levels, namely $35 \mathrm{MW}, 75 \mathrm{MW}$, and $115 \mathrm{MW}$ with probability of occurrence 0.5 , 0.3 and 0.2 respectively.

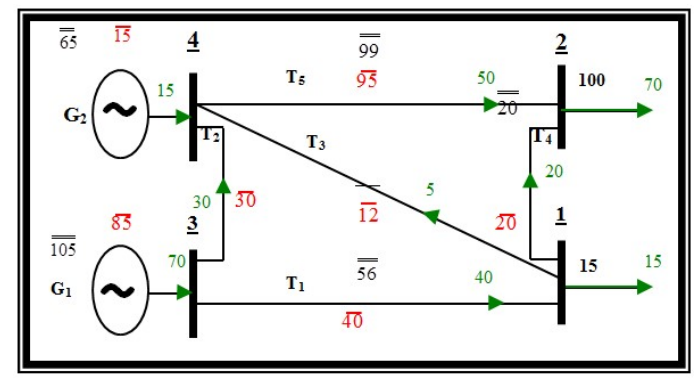

Fig. 4. A 4-bus sample power system

The combined generation/transmission state probability table associated with the generation and transmission capacities of this system, along with the reliability and performance quality indices $L N S, Q_{g 111}$ and $Q_{g 110}$ are shown in Table II for the $35 \mathrm{MW}$ load level. The rows of Table II represent various operating states. The first three columns of Table II represent the well-known generation Capacity Outage Probability Table (COPT) [20]. The next five columns (T1 to T5) represent the COPT for the transmission facilities of this system. Column $\left(\sum \bar{P}_{g}\right)$ is the total generation available capacities for each operating state shown in the Table. The column $\left(\sum P_{g}\right)$ represents the actual total generation as calculated from the solution of the Master Linear Program for various operating states. Similarly, the next three columns represent the associated values of the indices LNS and $Q_{g 111}$ and $Q_{g 110}$ respectively. When these values are multiplied by the operating state probability shown in the next column, they produce the expected values of these indices as outlined in the last three columns of the Table, namely eLNS and $e Q g 111$ and $e Q g 110$. Using the generation state availabilities shown in Table II, the discrete probability density functions of various capacities and indices can be evaluated and displayed. These discrete density functions show the overall probabilities of occurrence associated with given capacities or indices. For example, the probability density function of the generation capacity (state probabilities) for this system are depicted in Figure 5.

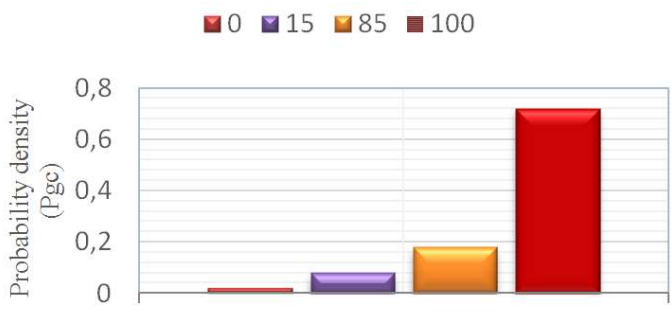

Generation Capacity Pgc (MW)

Fig. 5. Probability density of generation capacity for system
Figure 6 shows the probability density of the Utilized Generation Capacity $\left(Q_{g 111}\right)$ at $75 \mathrm{MW}$ load, while Figure 7 shows the probability density of Bottled Generation Capacity $\left(Q_{g 110}\right)$ at $115 \mathrm{MW}$ load and the probability density of Surplus Generation Capacity $\left(Q_{g 011}\right)$ for this system at $35 \mathrm{MW}$ load is shown in Figure 8. Table III summarizes the expected values of various performance quality and reliability indices for this system.

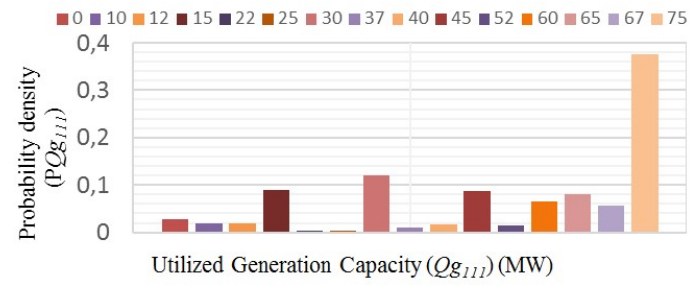

Fig. 6. Probability density of $Q_{g 111}$ for a system at $75 \mathrm{MW}$ load

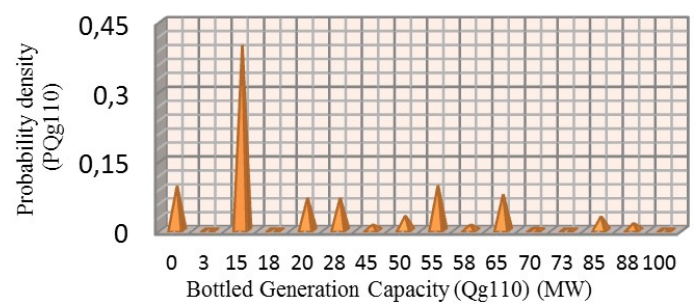

Fig. 7. Probability density of $Q_{g 110}$ for a system at $115 \mathrm{MW}$ load

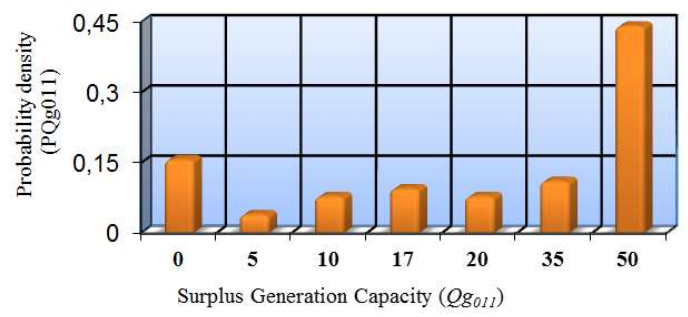

Fig. 8. Probability density of $Q_{g 011}$ for a system at $35 \mathrm{MW}$ load

The discrete probabilities of Figure 7 for $Q_{g 110}$ at $115 \mathrm{MW}$ load reveal that the highest probability is associated with generation bottling of $15 \mathrm{MW}$ and not with zero as would be expected for well-designed systems. This indicates a general weakness (shortage) in the installed transmission facilities as compared with the existing generation capacities and required load. The discrete probabilities of Figure 8 for $Q_{g 011}$ show two distinct high probabilities of occurrence at both 0 and $50 \mathrm{MW}$ surplus levels. This is mainly due to the fact that most of the main facilities (generators and transmission lines) are available (outage does not occur) which means that the network could deliver all the available generation (not needed) to the load side. On the other hand, at $Q_{g 011}=0$, most of the main facilities (generators and transmission lines) are not available (outage), especially G1, T1 and T2, meaning that the network either doesn't has the capacity of generation over the required load or it is incapable to deliver the available generation (not needed) through transmission lines to the load side. In this regard, the maximum value of the probability density of the $Q_{g 111}$ for the system occurs at $75 \mathrm{MW}$ load level as shown in Figure 8. 
TABLE II. PERFORMANCE QUALITY INDICES FOR THE TEST SYSTEM AT 35MW LOAD

\begin{tabular}{|c|c|c|c|c|c|c|c|c|c|c|c|c|c|c|c|c|}
\hline$\#$ & $\mathbf{G}_{1}$ & $\mathbf{G}_{2}$ & $\mathbf{T}_{1}$ & $\mathbf{T}_{2}$ & $\mathbf{T}_{3}$ & $\mathbf{T}_{4}$ & $\mathbf{T}_{5}$ & $\sum \overline{P_{g}}$ & $\sum P_{g}$ & $L N S$ & $Q_{g 111}$ & $Q_{g 110}$ & Probability & $e L N S$ & $e Q_{g 111}$ & $e Q_{g 110}$ \\
\hline 1 & 1 & 1 & 1 & 1 & 1 & 1 & 1 & 100 & 85 & 0 & 35 & 0 & 0.319468 & 0 & 11.18137 & 0 \\
\hline 2 & 1 & 1 & 1 & 1 & 1 & 1 & 0 & 100 & 52 & 10 & 25 & 10 & 0.056377 & 0.563767 & 1.409417 & 0.563767 \\
\hline 3 & 1 & 1 & 1 & 1 & 1 & 0 & 1 & 100 & 85 & 0 & 35 & 0 & 0.056377 & 0 & 1.973184 & 0 \\
\hline 4 & 1 & 1 & 1 & 1 & 1 & 0 & 0 & 100 & 52 & 30 & 5 & 30 & 0.009949 & 0.298465 & 0.049744 & 0.298465 \\
\hline 5 & 1 & 1 & 1 & 1 & 0 & 1 & 1 & 100 & 85 & 0 & 35 & 0 & 0.056377 & 0 & 1.973184 & 0 \\
\hline 6 & 1 & 1 & 1 & 1 & 0 & 1 & 0 & 100 & 40 & 10 & 25 & 10 & 0.009949 & $\begin{array}{l}0.099488 \\
\end{array}$ & 0.248721 & 0.099488 \\
\hline 7 & 1 & 1 & 1 & 1 & 0 & 0 & 1 & 100 & 85 & 0 & 35 & 0 & 0.009949 & 0 & 0.348209 & 0 \\
\hline 8 & 1 & 1 & 1 & 1 & 0 & 0 & 0 & 100 & 40 & 30 & 5 & 30 & 0.001756 & 0.05267 & 0.008778 & 0.05267 \\
\hline 9 & 1 & 1 & 1 & 0 & 1 & 1 & 1 & 100 & 55 & 0 & 35 & 0 & 0.056377 & 0 & 1.973184 & 0 \\
\hline 10 & 1 & 1 & 1 & 0 & 1 & 1 & 0 & 100 & 52 & 10 & 25 & 10 & 0.009949 & 0.099488 & 0.248721 & 0.099488 \\
\hline 11 & 1 & 1 & 1 & 0 & 1 & 0 & 1 & 100 & 55 & 3 & 32 & 3 & 0.009949 & 0.029846 & 0.318362 & 0.029846 \\
\hline 12 & 1 & 1 & 1 & 0 & 1 & 0 & 0 & 100 & 52 & 30 & 5 & 30 & 0.001756 & $\begin{array}{l}0.05267 \\
\end{array}$ & 0.008778 & 0.05267 \\
\hline 13 & 1 & 1 & 1 & 0 & 0 & 1 & 1 & 100 & 55 & 0 & 35 & 0 & 0.009949 & 0 & 0.348209 & 0 \\
\hline 14 & 1 & 1 & 1 & 0 & 0 & 1 & 0 & 100 & 40 & 10 & 25 & 10 & 0.001756 & 0.017557 & 0.043892 & 0.017557 \\
\hline 15 & 1 & 1 & 1 & 0 & 0 & 0 & 1 & 100 & 55 & 15 & 20 & 15 & 0.001756 & 0.026335 & 0.035114 & 0.026335 \\
\hline 16 & 1 & 1 & 1 & 0 & 0 & 0 & 0 & 100 & 40 & 30 & 5 & 30 & 0.00031 & 0.009295 & 0.001549 & 0.009295 \\
\hline 17 & 1 & 1 & 0 & 1 & 1 & 1 & 1 & 100 & 45 & 0 & 35 & 0 & 0.056377 & 0 & 1.973184 & 0 \\
\hline 18 & 1 & 1 & 0 & 1 & 1 & 1 & 0 & 100 & 12 & 23 & 12 & 23 & 0.009949 & 0.228823 & 0.119386 & 0.228823 \\
\hline 19 & 1 & 1 & 0 & 1 & 1 & 0 & 1 & 100 & 45 & 0 & 35 & 0 & 0.009949 & 0 & 0.348209 & 0 \\
\hline 20 & 1 & 1 & 0 & 1 & 1 & 0 & 0 & 100 & 12 & 30 & 5 & 30 & 0.001756 & 0.05267 & 0.008778 & 0.05267 \\
\hline 21 & 1 & 1 & 0 & 1 & 0 & 1 & 1 & 100 & 45 & 0 & 35 & 0 & 0.009949 & 0 & 0.348209 & 0 \\
\hline 22 & 1 & 1 & 0 & 1 & 0 & 1 & 0 & 100 & 0 & 35 & 0 & 35 & 0.001756 & 0.061449 & 0 & 0.061449 \\
\hline 23 & 1 & 1 & 0 & 1 & 0 & 0 & 1 & 100 & 45 & 5 & 30 & 5 & 0.001756 & 0.008778 & 0.05267 & 0.008778 \\
\hline 24 & 1 & 1 & 0 & 1 & 0 & 0 & 0 & 100 & 0 & 35 & 0 & 35 & 0.00031 & 0.010844 & 0 & 0.010844 \\
\hline 25 & 1 & 1 & 0 & 0 & 1 & 1 & 1 & 100 & 15 & 20 & 15 & 20 & 0.009949 & 0.198977 & 0.149232 & 0.198977 \\
\hline 26 & 1 & 1 & 0 & 0 & 1 & 1 & 0 & 100 & 12 & 23 & 12 & 23 & 0.001756 & 0.040381 & 0.021068 & 0.040381 \\
\hline 27 & 1 & 1 & 0 & 0 & 1 & 0 & 1 & 100 & 15 & 20 & 15 & 20 & 0.001756 & 0.035114 & 0.026335 & 0.035114 \\
\hline 28 & 1 & 1 & 0 & 0 & 1 & 0 & 0 & 100 & 12 & 30 & 5 & 30 & 0.00031 & 0.009295 & 0.001549 & 0.009295 \\
\hline 29 & 1 & 1 & 0 & 0 & 0 & 1 & 1 & 100 & 15 & 20 & 15 & 20 & 0.001756 & 0.035114 & 0.026335 & 0.035114 \\
\hline 30 & 1 & 1 & 0 & 0 & 0 & 1 & 0 & 100 & 0 & 35 & 0 & 35 & 0.00031 & 0.010844 & 0 & 0.010844 \\
\hline 31 & 1 & 1 & 0 & 0 & 0 & 0 & 1 & 100 & 15 & 20 & 15 & 20 & 0.00031 & 0.006197 & 0.004647 & 0.006197 \\
\hline 32 & 1 & 1 & 0 & 0 & 0 & 0 & 0 & 100 & 0 & 35 & 0 & 35 & $5.47 \mathrm{E}-05$ & 0.001914 & 0 & 0.001914 \\
\hline 33 & 1 & 0 & 1 & 1 & 1 & 1 & 1 & 85 & 70 & 0 & 35 & 0 & 0.079867 & 0 & 2.795343 & 0 \\
\hline 34 & 1 & 0 & 1 & 1 & 1 & 1 & 0 & 85 & 52 & 10 & 25 & 10 & 0.014094 & 0.140942 & 0.352354 & 0.140942 \\
\hline 35 & 1 & 0 & 1 & 1 & 1 & 0 & 1 & 85 & 70 & 0 & 35 & 0 & 0.014094 & 0 & 0.493296 & 0 \\
\hline 36 & 1 & 0 & 1 & 1 & 1 & 0 & 0 & 85 & 52 & 30 & 5 & 30 & 0.002487 & 0.074616 & 0.012436 & 0.074616 \\
\hline 37 & 1 & 0 & 1 & 1 & 0 & 1 & 1 & 85 & 70 & 0 & 35 & 0 & 0.014094 & 0 & 0.493296 & 0 \\
\hline 38 & 1 & 0 & 1 & 1 & 0 & 1 & 0 & 85 & 40 & 10 & 25 & 10 & 0.002487 & 0.024872 & 0.06218 & 0.024872 \\
\hline 39 & 1 & 0 & 1 & 1 & 0 & 0 & 1 & 85 & 70 & 0 & 35 & 0 & 0.002487 & 0 & 0.087052 & 0 \\
\hline 40 & 1 & 0 & 1 & 1 & 0 & 0 & 0 & 85 & 40 & 30 & 5 & 30 & 0.000439 & 0.013168 & 0.002195 & 0.013168 \\
\hline 41 & 1 & 0 & 1 & 0 & 1 & 1 & 1 & 85 & 40 & 0 & 35 & 0 & 0.014094 & 0 & 0.493296 & 0 \\
\hline 42 & 1 & 0 & 1 & 0 & 1 & 1 & 0 & 85 & 40 & 10 & 25 & 10 & 0.002487 & 0.024872 & 0.06218 & 0.024872 \\
\hline 43 & 1 & 0 & 1 & 0 & 1 & 0 & 1 & 85 & 40 & 18 & 17 & 18 & 0.002487 & 0.04477 & 0.042283 & 0.04477 \\
\hline 44 & 1 & 0 & 1 & 0 & 1 & 0 & 0 & 85 & 40 & 30 & 5 & 30 & 0.000439 & 0.013168 & 0.002195 & 0.013168 \\
\hline 45 & 1 & 0 & 1 & 0 & 0 & 1 & 1 & 85 & 40 & 10 & 25 & 10 & 0.002487 & 0.024872 & 0.06218 & 0.024872 \\
\hline 46 & 1 & 0 & 1 & 0 & 0 & 1 & 0 & 85 & 40 & 10 & 25 & 10 & 0.000439 & 0.004389 & $\begin{array}{l}0.010973 \\
\end{array}$ & 0.004389 \\
\hline 47 & 1 & 0 & 1 & 0 & 0 & 0 & 1 & 85 & 40 & 30 & 5 & 30 & 0.000439 & 0.013168 & 0.002195 & 0.013168 \\
\hline 48 & 1 & 0 & 1 & 0 & 0 & 0 & 0 & 85 & 40 & 30 & 5 & 30 & $7.75 \mathrm{E}-05$ & 0.002324 & 0.000387 & 0.002324 \\
\hline 49 & 1 & 0 & 0 & 1 & 1 & 1 & 1 & 85 & 30 & 5 & 30 & 5 & 0.014094 & 0.070471 & 0.422825 & 0.070471 \\
\hline 50 & 1 & 0 & 0 & 1 & 1 & 1 & 0 & 85 & 12 & 35 & 0 & 35 & 0.002487 & 0.087052 & 0 & 0.087052 \\
\hline 51 & 1 & 0 & 0 & 1 & 1 & 0 & 1 & 85 & 30 & 5 & 30 & 5 & 0.002487 & 0.012436 & 0.074616 & 0.012436 \\
\hline 52 & 1 & 0 & 0 & 1 & 1 & 0 & 0 & 85 & 12 & 30 & 5 & 30 & 0.000439 & 0.013168 & 0.002195 & 0.013168 \\
\hline 53 & 1 & 0 & 0 & 1 & 0 & 1 & 1 & 85 & 30 & 5 & 30 & 5 & 0.002487 & 0.012436 & 0.074616 & 0.012436 \\
\hline 54 & 1 & 0 & 0 & 1 & 0 & 1 & 0 & 85 & 0 & 35 & 0 & 35 & 0.000439 & 0.015362 & 0 & 0.015362 \\
\hline 55 & 1 & 0 & 0 & 1 & 0 & 0 & 1 & 85 & 30 & 5 & 30 & 5 & 0.000439 & 0.002195 & 0.013168 & 0.002195 \\
\hline 56 & 1 & 0 & 0 & 1 & 0 & 0 & 0 & 85 & 0 & 35 & 0 & 35 & $7.75 \mathrm{E}-05$ & 0.002711 & 0 & 0.002711 \\
\hline 57 & 1 & 0 & 0 & 0 & 1 & 1 & 1 & 85 & 0 & 35 & 0 & 35 & 0.002487 & 0.087052 & 0 & 0.087052 \\
\hline
\end{tabular}

\section{CONCLUSIONS}

A general and coherent formulation is presented which can be used to account for the randomness associated with the load level and the availability of generation and transmission capacities. The general probability formulation can be used to calculate various reliability indices and quality measures. The paper describes the new approach for computing the probabilistic evaluation (expected value) of the reliability indices and performance quality measures and presents 
illustrative applications. The expected values of reliability indices, such as the Expected Load Not-Served (eLNS) and the expected values of performance quality indices: Utilized Generation Capacity $\left(e Q_{g 111}\right)$, Bottled Generation Capacity $\left(e Q_{g 110}\right)$, Shortfall Generation Capacity $\left(e Q_{g 101}\right)$, Deficit Generation Capacity $\left(e Q_{g 100}\right)$, Surplus Generation Capacity $\left(e Q_{g 011}\right)$, Redundant Generation Capacity $\left(e Q_{g 010}\right)$, Spared Generation Capacity $\left(e Q_{g 001}\right)$, and Saved Generation Capacity $\left(e Q_{g 000}\right)$ are calculated in this paper with the established flow pattern, based on the solution of the basic linear program .

TABLE III. EXPECTED VALUE OF RELIABILITY AND QUALITY PERFOMANCE INDICES

\begin{tabular}{|c|c|c|c|c|c|}
\hline Index & $\boldsymbol{e} \mathbf{L N S}$ & $\boldsymbol{e} \boldsymbol{Q}_{\boldsymbol{g} \mathbf{1 1 1}}$ & $\boldsymbol{e} \boldsymbol{Q}_{\boldsymbol{g} \mathbf{1 1 0}}$ & $\boldsymbol{e} \boldsymbol{Q}_{\boldsymbol{g} \mathbf{1 0 1}}$ & $\boldsymbol{e} \boldsymbol{Q}_{g \mathbf{1 0 0}}$ \\
\hline Value (MW) & 21.44 & 43.56 & 12.90 & 6.85 & 0.99 \\
\hline Index & $\boldsymbol{e} \boldsymbol{Q}_{g \mathbf{0 1 1}}$ & $\boldsymbol{e} \boldsymbol{Q}_{\boldsymbol{g} \mathbf{1 0}}$ & $\boldsymbol{e} \boldsymbol{Q}_{\boldsymbol{g} \mathbf{0 0 1}}$ & $\boldsymbol{e} \boldsymbol{Q}_{\boldsymbol{g} \mathbf{0 0 0}}$ & \\
\hline Value (MW) & 15.11 & 17.14 & 34.03 & 25.36 & \\
\hline
\end{tabular}

The general framework and formulation introduced in this paper can be applied to practical power systems. Also, it can be applied to a system under normal operation or subject to contingencies with certain or random occurrences. In this paper, the optimization technique based on three metaphors (dimensions) representing the relationship between available generation capacities and required demand levels was utilized. The novel formulation presented in this paper can accommodate the randomness associated with the load level as well as the availability of generation and transmission capacities, in this case, the expected values of reliability indices and the expected values of performance quality measures.

\section{ACKNOWLEDGMENT}

This work was supported by the University of Ha'il.

\section{REFERENCES}

[1] R. Billinton, E. Khan, "A security based approach to composite power system reliability evaluation", IEEE Transactions on Power Systems, Vol. 7, No. 1, pp. 65-72, 1992

[2] M. A. El-Kady, B. M. Alshammary, "A practical framework for reliability and quality assessment of power systems", Journal of Energy and Power Engineering, Vol. 3, No. 4, pp. 499-507, 2011

[3] H. Jun, Y. Zhao, P. Lindsay, P. Kit, "Flexible transmission expansion planning with uncertainties in an electricity market", IEEE Transactions on Power Systems, Vol. 24, No. 1, pp. 479-488, 2009

[4] S. D Torre, A. J. Conejo, J. Contreras, "Transmission expansion planning in electricity markets", IEEE Transactions on Power Systems, Vol. 23, No. 1, pp. 238-248, 2008

[5] B. M. Alshammari, "Assessment of reliability and quality performance using impact of shortfall generation capacity index on power systems", Engineering, Technology \& Applied Science Research, Vol. 9, No. 6, pp. 4937-4941, 2019

[6] D. O. Koval, A. A. Chowdhury, "Outage data concepts for generation and transmission equipment", IEEE Power Engineering Society General Meeting, Montreal, Canada, June 18-22, 2006

[7] M. A. El-Kady, B. A. Alaskar, A. M. Shaalan, B. M. Al-Shammri, "Composite reliability and quality assessment of interconnected power systems", International Journal for Computation and Mathematic in Electrical and Electronic Engineering, Vol. 26, No. 1, Article ID SSD05PES-12, 2007

[8] B. M. Alshammari, M. A. El-Kady, Y. A. Al-Turki, "Computer-aided optimization of power system performance quality indices", 3rd Global Conference on Power Control and Optimization, Gold Coast, Australia, February 2-4, 2010
[9] B. M. Alshammari, M. A. El-Kady, Y. A. Al-Turki, "Power system performance quality indices", European Transactions on Electrical Power, Vol. 21, No. 5, pp. 1704-1710, 2011

[10] O. Kahouli, B. Ashammari., K. Sebaa, M. Jebali, H. Hadj Abdallah "Type-2 fuzzy logic controller based PSS for large scale power systems stability", Engineering, Technology \& Applied Science Research, Vol. 8, No. 5, pp. 3380-3386, 2018

[11] P. Jirutitijaroen, C. Singh, "Reliability constrained multi-area adequacy planning using stochastic programming with sample-average approximations", IEEE Transactions on Power Systems, Vol. 23, No. 2, pp. $405-513,2008$

[12] R. Billinton, D. Huang, "Effects of load forecast uncertainty on bulk electric system reliability evaluation", IEEE Transactions on Power Systems, Vol. 23, No. 2, pp. 418-425, 2008

[13] M. de Jong, G. Papaefthymiou, P. Palensky, "A framework for incorporation of infeed uncertainty in power system risk-based security assessment", IEEE Transactions on Power Systems, Vol. 33, No. 1, pp. 613-621, 2018

[14] B. M. Alshammari, M. A. El-Kady, "Probabilistic assessment of power system performance quality", Journal of Energy and Power Engineering, Vol. 4, No. 5, pp. 372-379, 2012

[15] S. Gope, A. K. Goswami, P. K. Tiwari, "Transmission congestion management using a wind integrated compressed air energy storage system", Engineering, Technology \& Applied Science Research, Vol. 7, No. 4, pp. 1746-1752, 2017

[16] M. de Jong, G. Papaefthymiou, P. Palensky, "A framework for incorporation of infeed uncertainty in power system risk-based security assessment", IEEE Transactions on Power Systems, Vol. 33, No. 1, pp. 613-621, 2018

[17] B. M. Alshammari. "Evaluation of power system reliability levels for (N-1) outage contingency", International Journal of Advanced and Applied Sciences, Vol. 6, No. 11, pp. 68-74, 2019

[18] E. Tomasson, L. Soder, "Improved importance sampling for reliability evaluation of composite power systems", IEEE Transactions on Power Systems, Vol. 32, No. 3, pp. 2426-2434, 2017

[19] M. de Jong, G. Papaefthymiou, P. Palensky, "A framework for incorporation of infeed uncertainty in power system risk-based security assessment”, IEEE Transactions on Power Systems, Vol. 33, No. 1, pp. 613-621, 2018

[20] R. Billinton, R. N. Allan, Reliability assessment of large electric power systems, Kluwer Acadmic Publisher, 1988 